Vol. 1, No. 2, 2016

\title{
CHARACTERISTIC EXPRESS CHECKING OF ADMITTANCE MEASUREMENT UNITS FOR CYBER-PHYSICAL SYSTEMS
}

\author{
Vasyl Yatsuk, Yevhen Pokhodylo, Tetiana Bubela, Yurii Yatsuk \\ Lviv Polytechnic National University, 12, Bandera str., Lviv, 79013, Ukraine. \\ Authors e-mail: Yatsuk.vasyl@gmail.com
}

Submitted on 02.12.2016

(C) Yatsuk V., Pokhodylo Ye., Bubela T., Yatsuk Yu., 2016

Abstract. Measurement of RLC-parameters of objects non-electric nature (for example, biological, agricultural, environmental, etc.) with high metrological characteristics is an essential element for building a cyber-physical systems analytical purposes. The reliability of the measurement results of the equipment can be provided only by periodic metrological control over the exploitation conditions. Traditionally analytical RLC-meters are calibrated by means of standard samples and it leads to significant problems in their metrological assurance and complicates whole procedure. It is suggested to make operating check of admittance measurement units metrological characteristics by use of intelligent devices based on code-controlled measures. Critical analysis of available approaches for measures creation was carried out and an admittance codecontrolled measure scheme based on active simulators was designed. The mathematic model for measuring transfer function, analytical devices calibration process and advantages of the operating check based code-controlled measure was presented too.

Index Terms - Code-controlled measures, cyber-physical systems, measurement channels, operative check of metrological characteristics, remote auto calibration, express control, physical-chemical measurements, codecontrolled measure, admittance, metrological characteristics, analytical devices.

\section{INTRODUCTION}

Creation of cyber-physical systems (CPS) for parameter monitoring and environment quality management would contribute to a significant improvement of the ecological situation, sustainable use of natural resources, including water and soil. CPS would streamline the collection and analysis of prognostic parameters of land (soil) in real time and with linking to spatial coordinates and improve their certification process and technical supervision over the use of certified soil. Forming the information base of state monitoring of soil contamination at the CPS would provide the appropriate level of management.

To optimize the process of gathering information about the quality indices (QI) of soil, it is appropriate to use the admittance method [1,2], under which nonelectric nature object (soil), placed in the electric circuit of alternating current, is seen as complicated bipolar object. Dependency parameters of this object contain information about relevant physicochemical properties of the controlled object. As a result of measuring RLC-parameters of bipolar object, relationship between electric and relevant physicochemical characteristics will be set up. This method would be best to control following important integrated soil QI as the level of acidity, salinity, humidity and more.

This will encourage admittance soil maps creation and their use in real time, which will reduce the costs of inspection and will provide a more objective and representative information on changing soil properties in space and heterogeneity of soil parameters compared with investigations, performed only by classical methods. Responsiveness to diverse plant growth conditions will improve yields at the fixed production costs and / or reduce those costs without reducing yield. Work of mobile unit for admittance mapping can be based on capacitive method.

For example, the electrodes can be made in the form of discs (they can be attached to a vehicle moving at a constant speed on the field), which are in contact with the soil and this will enable to determine the admittance in several layers of soil at different depths and at specific times.

Thus, the amount of data will be much more dense than with traditional data collection. Global Positioning Devices (GPS) will allow to get territorial information distribution. Admittance maps illustrate contrasting soil zone. Although the absolute value of admittance will increase with increasing soil moisture, but the relative values remain consistently adequate through regular intervals.

In order to ensure a high degree of reliability of RLCmeasurement results for analytical purpose on the base of CPS it is essential to control the metrological characteristics of measuring channels in place of exploitation $[3,4]$.

\section{THE CONCEPT OF METROLOGICAL CHARACTERISTICS EXPRESS CONTROL OF ADMITTANCE MEASUREMENT UNIT'S ANALYTICAL PURPOSE}

Therefore, the measurement channel calibration of analytical purpose RLC-meters is proposed to carry out using intelligent devices based on code-controlled measures. To do this, first of all, you should prepare series of standard samples, which will act as a 
concentration measure of controlled object in the whole possible range of changes. On the basis of results measurements massif in the given frequency band of impedance (admittance) electrical parameters of this standard samples, which is the certain object concentration measure, by precision measure RLC-parameters and finally work up this results. It is conducted by digital codes matrix $\mathrm{Ni}$ formation for descriptive measurer $\mathrm{ZM}$, that correspond to measured parameters of the given object (Fig. 1).

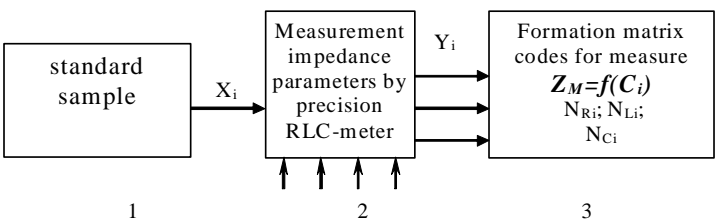

Fig. 1. General structure of electrical measure parameters determination for analytic measurement device calibration

Hereinafter, metrological characteristic control of admittance measurement is worthwhile to carry out by substitution method or by differential or compensatory methods of resistance measurement [5, 6] (Fig. 2).

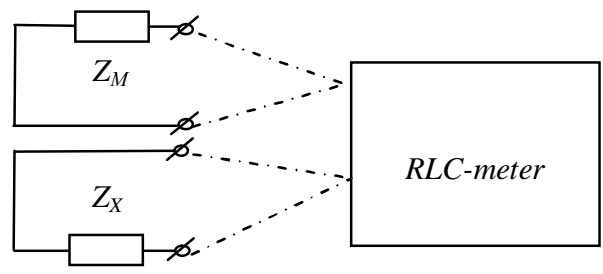

Fig. 2. Scheme of substitution method

For example, at impedance measurement $Z_{X}$ by substitution method of impedance bridge meters the measurement result can be presented as [7]

$$
Z_{X}=Z_{M} \cdot \frac{Z_{c m p X}}{Z_{c m p M}},
$$

where $Z_{c m p X}$ - comparison arm resistance value at inclusion in resistance scheme $Z_{X} ; Z_{c m p M}$ - comparison arm resistance value at inclusion in resistance scheme $Z_{M}$.

On condition that comparison arm resistance errors at impedance measurement $Z_{X}$ and $Z_{M}$ are approximately the same $\delta_{c m p X} \approx \delta_{c m p M}$, will have that impedance measurement errors $Z_{X}$ be almost equal the sum of measure error and two discreteness $\delta_{d s t}$ errors $\delta_{c m p X}=\delta_{c m p M}+2 \delta_{d s t}$.

\section{THE PRACTICAL IMPLEMENTATION OF CODE-CONTROLLED ADMITTANCE (IMPEDANCE) MEASURE}

\section{A. Code-controlled boxes}

Usually, system substitution scheme measuring converter and the checking object that is parallel to connection of resistance and capacitance (Fig. 3). Multivalued measures of
$\mathrm{RC}$-parameters can be realized as code-controlled boxes, since complex resistance has a additivity property [7, 8]. The design principle realization is usually used in codecontrolled resistance boxes in which scalable resistors and electronic shunt keys are parallel connection and such code-controlled box output resistance with residual keys parameters is presented in ratio:

$$
\begin{aligned}
R_{12} & =\sum_{i=1}^{4} a_{i} R_{i}+\sum_{i=1}^{4}\left(1-a_{i}\right) \frac{r_{k i}}{1+r_{k i} / R_{i}}+\sum_{i=1}^{4} a_{i} \frac{R_{i}}{1+R_{i} / R_{p i}}+\sum_{i=1}^{4}\left(1-a_{i}\right) \frac{e_{k i}}{I_{12}}+\ldots+ \\
& +\sum_{j=1}^{4} a_{j} R_{j}+\sum_{j=1}^{4}\left(1-a_{j}\right) \frac{r_{k j}}{1+r_{k j} / R_{j}}+\sum_{i=1}^{4} a_{j} \frac{R_{j}}{1+R_{j} / R_{p j}}+\sum_{j=1}^{4}\left(1-a_{j}\right) \frac{e_{k j}}{I_{12}}
\end{aligned}
$$

where $r_{k}, e_{k i}, r_{k J}, e_{k J}-$ resistance of $i$ or ${ }_{\mathrm{j}}$ closed key and residual voltage on them respectively; $R_{p i}, R_{p j}-$ resistance of $i$ or $j$ open key respectively; $R_{i}, R_{j}-$ scalable resistances of $i$ or $j$ code-controlled tetrad; $a_{i}$ interconnect coefficient $\left(a_{i}=0\right.$ is a key is closed, respectively, turned off resistance with replicable resistance measure, $a_{i}=1$ otherwise); $I_{12}$ - the current that flows between clamps 1 and 2 of resistance measure.

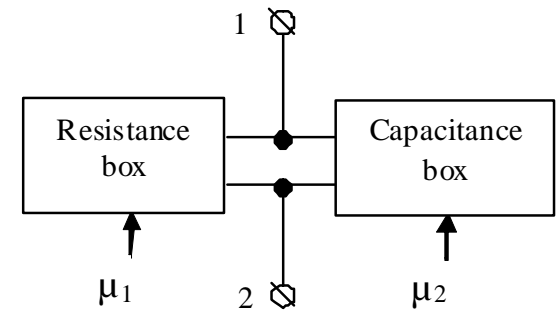

Fig. 3. General scheme of switching admittance code-controlled measure

In code-controlled capacity boxes scalable elements are connected with terminal leads parallel by summing capacity of enabled condenser. After simple transformations on decomposition of expression denominator of capacitive conductivity parallel parts in a row and neglecting components of the second order and above of smallness and will get such expression for the value evaluation of installed capacitive conductivity $Y_{12}$ box

$$
\begin{aligned}
Y_{12} & \cong \sum_{i=1}^{m} a_{i} j \omega C_{i}-\sum_{i=1}^{m} a_{i} j \omega C_{i} \frac{e_{k i}}{U_{12}}-\sum_{i=1}^{m} a_{i} j \omega C_{i} \frac{z_{k i}}{z_{p i}}+ \\
& +\sum_{i=1}^{m}\left(1-a_{i}\right) \frac{1}{z_{p i}}+j \omega C_{\Pi}
\end{aligned}
$$

where $C_{i}$ - scalable capacity of $i$ order; $e_{k i}, z_{k i}, z_{p i}-$ respectively residual voltage, resistance of closed and open $i$ key; m - orders quantity of capacity box; $C_{\Pi}-$ parasitic capacitance between clamps 1 and 2 of capacity box; $\omega$ - circular frequency.

Previous analysis shows that in multivalued measures of RC-parameters residual parameters of switching elements make a significant error, that limit their accuracy and discreteness of impedance reproduction. 


\section{B. Impedance transformer measures}

One of the perspective ways to solve this problem is a use of impedance transformer measures where the equivalent resistance is formed by means of full resistance transformation of exemplary element by transformer converters in equivalent resistance. It resistance value is proportional to transformation ratio 9 , 10]. Use of such devices allows expanding the range of nominal measures measure (to small impedance) in 100-1000 times. The easiest linear transformer impedance measure contains an exemplary element of full resistance $\mathrm{Z}_{0}$ and transformer $\mathrm{T}$, which serves as scalable converter (Fig. 4). Output terminals of primary winding $\mathrm{T}$ are connected to current measure clamps 1,2 , and to output terminals of secondary winding $\mathrm{T}$ current output terminals of exemplary elements $Z_{0}$. Potential output terminals of this element are connected to potential clamps 3,4 of the device. As value $\mathrm{Z}_{0}$ elements of any reactivity (inductance, capacitance, and resistance) can be used with the random transformer scheme. Equivalent resistance of transformer measure is determined by the voltage ratio $\mathcal{L}_{34}$, formed on potential clamps 3,4 , to current $\alpha_{1}^{\alpha}$, that flows in its primary circle

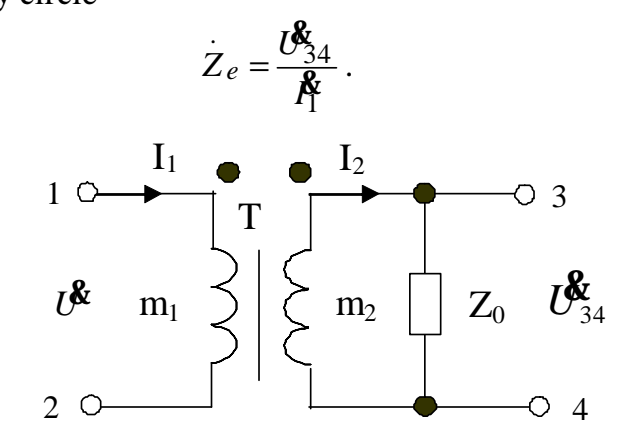

Fig. 4. Impedance transformer value scheme.

The transformation equation of simple linear transformer measure is as follows

$$
\dot{Z}_{e}=\dot{Z}_{0} \frac{m_{1}}{m_{2}}[1+\gamma]
$$

where $Z_{0}$ - full resistance of exemplary element; $m_{1}$, $\mathrm{m}_{2}$ - coil quantity of primary and secondary transformer windings $\mathrm{T}$ respectively; $\gamma$ - error of the device impedance transformation.

Error $\gamma$ is caused by influence of magnetizing current of transformer converter, whose presence break proportionality between the primary and secondary windings $T$ currents and causes an effect of secondary circle bridging of transformation measure (active resistance $R_{2}$ of secondary winding $T$ and exemplary element $\mathrm{Z}_{0}$ ) by inductive reactance $\mathrm{X}_{2}$ of secondary winding $T$. To correct values of this error compensating elements are used, that leads to significant complications of scheme-technical realization. Such measures will have relatively narrow frequency band, complex system of winding commutation and low accuracy [20,21].
Previous analysis shows that disadvantages of impedance code-controlled boxes influence residual parameters of commutation elements. The unit of junior order of code-controlled box of impedance is limited by resistance value of closed key, does not allow realizing box with discrete reproduction lower than $0,1 \mathrm{Ohm}$ at a frequency $50 \mathrm{~Hz}$, as resistance of closed key for best reed keys is $0,05 \mathrm{Ohm}$. The resistance of open key will greatly affect at reproduction of high values of impedance, as their values become proportionate. Frequency range of codecontrolled box of impedance is limited by spurious capacities of open key. Furthermore, the impedance code-controlled box should have predicted key protection from overvoltage at transient condition. In addition, the inertia of switching elements significantly limits action speed of impedance box.

\section{The simulators of multilink feedback based on operational amplifiers $M$}

In general, the simulator of reactance can be implemented based on one active element (operational amplifier OA) covered by feedback via exemplary reactive element (Fig. 5).

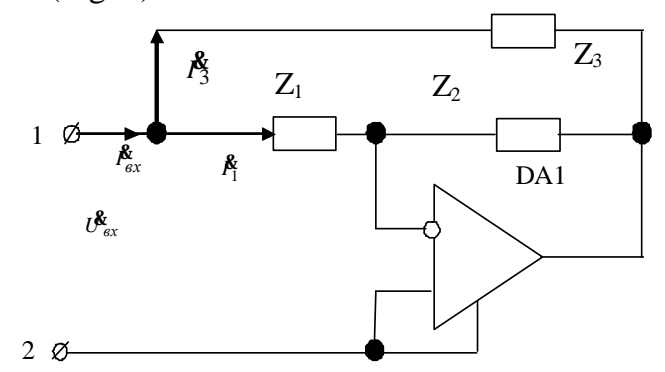

Fig. 5. Scheme of reactance simulator

Input conduction $Y_{I N}=I_{I N} / U_{I N}$ is determined as

$$
\dot{Y}_{B x}=\frac{1}{\delta}+\frac{1}{\delta}+\frac{\&}{\delta \cdot \delta},
$$

where $\mathcal{E}_{I N}, \mathcal{\&}_{I N}$ - input voltage and current measure respectively.

To compensate for parasitic input conduction $1 / \&$ and $1 / \&$ can be used negative resistance converters that are worth to switch on parallel to input clamps. Use of additional elements leads to measure error incensement, except, there can be used only resistance boxes on codecontrolled measures or capacitances that leads to mentioned above disadvantages. Finally, the structure can loss the stability due to the use of negative resistance converters during environmental changes.

\section{Reactance measures on potentially-stable elements}

Potentially-stable measures can be realized based on operational amplifiers with integrative potential feedback (Fig. 6).

The value of simulated resistance $Z_{i}$ for ideal operational amplifiers is determined as: 


$$
Z_{i}=\frac{U_{G x}^{\&}}{\&_{i}^{\alpha}}=\frac{U_{2}^{\&}}{\mathcal{E}_{i}^{\alpha}}=Z_{1} \frac{Z_{5}}{Z_{2}+Z_{5}} \cdot \frac{Z_{2}+Z_{6}}{Z_{2}} .
$$

If conditions $Z_{1}=R_{1}, Z_{2}=Z_{3}=R_{2}, Z_{5}=Z_{6}=1 / j \omega C$ meets, so the scheme Fig. 7 will reproduce the capacity $Z_{i}=\frac{R_{1}}{R_{2}} \cdot \frac{1}{j \omega C}$. In such code-controlled measure it can be used only code-controlled resistance box $R_{1}$, that will have inherent disadvantages of code-controlled resistance measures.

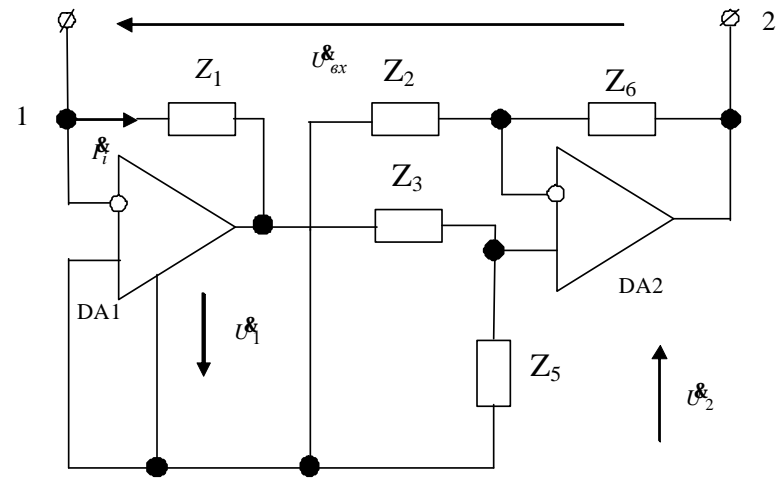

Fig. 6. Scheme of reactance potentially-stable measure E. Code-controlled measures of reactive resistance with loses

In practice RLC-measures of $\mathrm{L}$ and $\mathrm{C}$-elements are given by equivalent substitution scheme with loses. Code-controlled measures, in which the values of reactive and active resistance can be set, are required for AMD metrological check (Fig. 7) [8, 11].

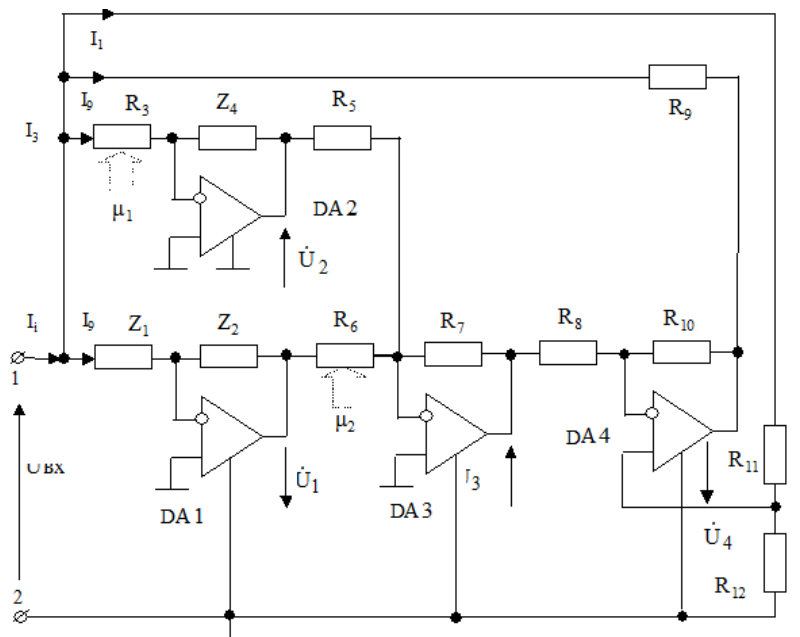

Fig. 7. Scheme of code-controlled box of complex values

The code-controlled box conductance value is determined as:

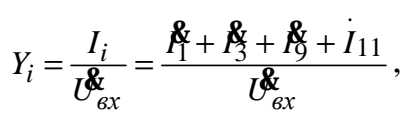

where $\AA_{1}^{\alpha}, \frac{\alpha}{2}, \frac{\alpha}{f_{1}}, \frac{\alpha}{\AA_{11}}$ - currents that flows through reactive resistance $Z_{1}$ and resistances $R_{3}, R_{9}, R_{11}$ accordingly.
Currents values determined by the ratio $\AA_{1}^{\alpha}=\frac{\mathcal{E}_{I N}}{Z_{1}}$; $\&_{3}=\frac{\mathcal{E}_{I N}}{R_{3}}, \quad \AA_{11}=\frac{\mathscr{\Psi}_{I N}}{R_{11}+R_{12}} ; \quad \&_{9}=\frac{U_{4}}{R_{9}} . \quad$ Complex conductance $Y_{i}$ simulated by the box can be presented as:

$$
\begin{aligned}
Y_{i}= & \frac{1}{Z_{1}}\left(1+\frac{Z_{2}}{R_{9}} \cdot \frac{R_{10}}{R_{8}} \cdot \frac{R_{7}}{R_{6}}\right)+\frac{1}{R_{3}}\left(1+\frac{R_{4}}{R_{9}} \cdot \frac{R_{10}}{R_{8}} \cdot \frac{R_{7}}{R_{5}}\right)+ \\
& +\frac{1}{R_{11}+R_{12}}\left(1-\frac{R_{12}}{R_{9}} \cdot \frac{R_{10}}{R_{8}}\right)
\end{aligned}
$$

From the expression (9) can be concluded, that codecontrolled box will imitate complex capacitive conductance under conditions $1-\frac{R_{12}}{R_{9}} \cdot \frac{R_{10}}{R_{8}}=0, Z_{2}=R$ and $Z_{l}=1 / j \omega C$.

The capacitive component value of the complex resistance can be settled by resistance change $R_{9}$, and an active one by resistance change $R_{3}$. If we specify that $Z_{1}=R, Z_{2}=1 / j \omega C$, $\frac{1}{R}+\frac{1}{R_{11}+R_{12}}\left(1-\frac{R_{12}}{R_{9}} \cdot \frac{R_{10}}{R_{8}}\right)=0$, so by means of given structure the inductive complex conductance can be reproduced. The value of complex resistance inductive component is set by code-change resistance $R_{6}$, and active by code-change $\mathrm{R}_{3}$ accordingly.

The peculiarity of analyzed structure (Fig. 7) [11] is fundamental disadvantages common to all resistance codecontrolled boxes. Use of capacity simulator-measures based on amplifiers with multi-loop feedback can lead to stability loss and significant limitation of frequency band due to use of differentiating feedback. Namely, present capacity and resistance calibrators (boxes) do not meet up-to-date AMD calibration requirements because of it have bad complex of metrological characteristics.

\section{DEVELOPED ADMITTANCE CODE-CONTROLLED MEASURE}

To improve technical characteristics, it is proposed to use simulators structures of RC-parameters that are consists of single-value measures, code-controlled voltage dividers (DAC) and active buffer cells (Fig. 8).

Input voltage $\mathrm{U}_{\mathrm{i}}$ through transducer OA11 or OA12 is fed to the entrance port of code-controlled voltage-ratio divider CVR11 or CVR12, which transfer ratio reach a value by internal code (by controller or by operator) in output of the second amplifier OA21 or OA22. Through calibrating capacitor $\mathrm{C}_{\mathrm{N}}$ (or resistor $\mathrm{R}_{\mathrm{N}}$ ) current flows $\mathrm{I}_{\mathrm{iC}}$ $\left(\mathrm{I}_{\mathrm{iR}}\right)$ under condition that input current OA11 or OA12 is negligible,

$$
I_{i c}=\frac{U_{i}-U_{2 C}}{X_{C_{N}}}=j \omega C_{N} \cdot(1-N) \cdot U_{i}
$$

or

$$
I_{i R}=\frac{U_{i}-U_{2 R}}{R_{N}}=(1-M) \cdot G_{N} \cdot U_{i},
$$

and scheme simulated admittance $Y$ will be determined as sum of active $Y_{R}$ and reactive $Y_{C}$ admittance components according to correlation: $Y=Y_{C}+Y_{R}$, where 


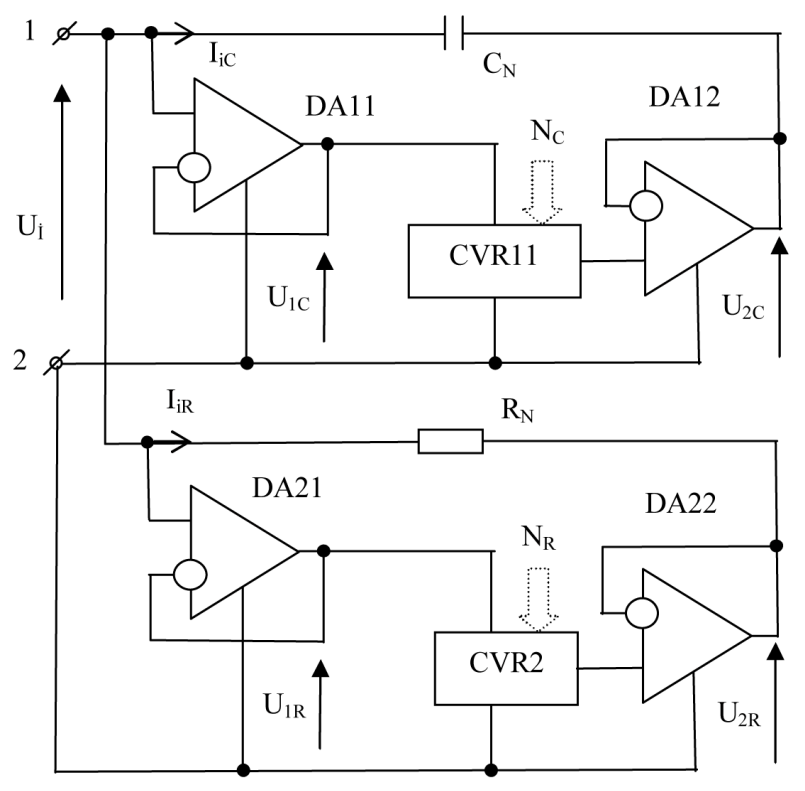

Fig. 8. Scheme of code-controlled measure of admittance simulator

$$
\begin{gathered}
Y_{C}=\frac{I_{i}}{U_{i}}=j \omega C_{N}(1-N)=j \omega C_{i}, \\
Y_{R}=\frac{I_{i}}{U_{i}}=(1-M) \cdot G_{N},
\end{gathered}
$$

where $\mathrm{C}_{\mathrm{N}}, X_{C N}=1 / j \omega C_{N}, G_{N}=1 / R_{N}$ - respectively, capacity and resistance of calibrating capacitor and conductivity of standard (reference) resistor; $\mathrm{U}_{2}=N \cdot U_{i}-$ voltage output of the second OA2; $N=N_{i} / N_{\max }$ - transfer ratio CVR1; $\mathrm{N}_{\mathrm{i}}, \mathrm{N}_{\max }-$ constant and maximum code value CVR1; $M=M_{i} / M_{\max }-$ transfer ratio CVR2; $\mathrm{M}_{\mathrm{i}}, \mathrm{M}_{\max }-$ constant and maximum code value CVR2.

\section{THE PERSPECTIVES OF PRACTICE IMPLEMENTATION OF PROPOSED METHOD FOR REMOTE ERRORS CORRECTION}

The use of code-controlled admittance value according to Fig. 8 will enable its miniaturization, therefore, inexpensive microelectronic implementation. Besides, the use of modern operational amplifiers can provide wide enough frequency band. Any voltage divider can be used fundamentally as CVR in considered structure, for example, integrated DAC, dividers based on alternating-current magnetic comparators or inductive divider. Under these conditions resulting error of resistance simulation can be determined only by errors of current specified resistor $R_{N}$ or capacitor $C_{N}$. Their small number and size enable the implementation of constructive code-controlled measure as compact plug-in assembly. It is required to carry out more frequent metrological check of such block to provide high temporal stability of impedance measures.

\section{CONCLUSIONS.}

The problem of ensuring the physical-chemical measurements unity is actualized in this paper. The immediate control of admittance measurement metrological characteristics units of non-electrical nature objects is one of the ways to solve this problem. For this purpose, it was suggested to carry out analytical RCmeters channel measurement calibration by means of intelligent devices based on code-controlled measures whose values are determined by standard samples. Substitution, compensation or differential methods can be used during calibration of resistance measures.

The ways of impedance code-controlled measures development were researched and the disadvantages were identified. A structure for practical realization of admittance (impedance) code-controlled measures based on active simulators was proposed.

\section{REFERENCES}

[1] Pokhodylo Ye., Stolyarchuk P. Methods of quality immitance control, Methods and devices for quality control. - 2003. No. 11. - P. 105-108. (in Ukrainian)

[2] T. Bubela, P. Stolyarchuk, M. Mykyychuk, O. Basalkevych, Admittance Method Application in the Maintenance of Ecomonitoring Information System for Soil Parameters, in Proceedings of the Conference on 'Intelligent Data Acquision and Advanced Computing System: Technology and Applications (IDAACS 2011)', Prague, Czech Republic, 15-17 September 2011, pp. 97-100.

[3] Yatsuk W., Wesełucha Z. Możliwości zwiększania niezawodności metrologicznej przyrządów pomiarowych z zastosowaniem wzorców miar sterowanych kodowo oraz nowoczesnych technologii informacyjnych // Przegląd Elektrotechniczny 2010, R. 86, nr 9, p. 80-83. (in Polish)

[4] T. Bubela, Ryabkova T. Operative checking algorithm for soil contamination. Proceedings of Lviv Polytechnic Computer systems and networks. - 2011. - № 688. - P. 30-34. (in Ukrainian)

[5] V. Yatsuk. Differential method of electric resistance measurement. - Measurement technic and metrology. - 2001. - No. 58. P. 32-37.

[6] Patent. 109387 Ukraine, МПК H03H 11/28. Impedance codecontrol measure / V. Yatsuk, T. Bubela, P. Stolyarchuk, M. Mykyychuk, Yu. Yatsuk.; claimer and owner Lviv Polytechnic National University. - No. a 201413672, claim. 19.12.2014; publ. 10.08.2015, Bul. No. 15.

[7] Ye. Polishchuk, M. Dorozhovets, V. Yatsuk. and al. Metrology and Measurement Technic: Hand-book; Under red. Ye. Polishchuk. - Lviv: "Beskyd-bit" Publish House, 2003. 544 p. (in Ukrainian)

[8] V. Yatsuk, R. Yanovych, Y. Yatsuk, V. Zdeb, Possibilities of Precision Ohmmeter Calibration in the Exploitation Condition, in Proceedings of the Conference on 'Intelligent Data Acquision and Advanced Computing System: Technology and Applications (IDAACS 2013)', Berlin, Germany, 12-14 September 2013, pp. 86-89.

[9] Patent 1566301 USSR, МКИ G 01 R 27/00. Transformer impedance measure / A. Vdovin, V. Karpenko, V. Kohut, V. Kozmenko, S. Makarenko, M. Surdu. - No. 4301315/24-09; claim. 27.08.1987; publ. 23.05.1990, Bul. № 19. (in Russian)

[10] Yu. Bratus, E. Budnicka. V. Karpenko. Transformer reference measure construction // Elements and schemes of electro measurement units and systems. - Kyjiv: Naukova dumka publ. house. - 1985. - P. 42-51.

[11] Imitator of complex conductivity boxes. Patent. 1218451 (USSR) // Yu. Bratus, E. Budnicka. V. Karpenko., S. Makarenko, Yu. Smolyar. - Claim. 03.01.84 No. 3685343/24-09; Publ. 15.03.86. Bul 10. - 1986. -3 p. 


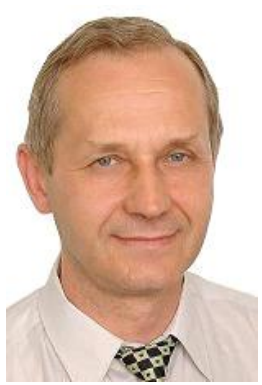

Prof. DSc. Vasyl Yatsuk

Professor of Lviv Polytechnic National University. He specializes in the field of measuring of electrical and non-electrical quantities and their metrological support. He is the author of over 170 scientific and methodical works, co-author of 4 textbooks. Participated in the development of serial production of digital devices in industrial enterprises.

Lviv Polytechnic National University 12, Bandera str., Lviv 79008, Ukraine e-mail:vyatsuk@polynet.lviv.ua.

\section{Prof. DSc. Yevgen Pokhodylo}

Professor of Lviv Polytechnic National University. He specializes in the field of measuring of electrical and non-electrical quantities. $\mathrm{He}$ is the author of over 200 scientific and methodical works, co-author of 3 textbooks. Participated in the development of serial production of digital devices in industrial enterprises.

Lviv Polytechnic National University 12, Bandera str., Lviv 79008, Ukraine e-mail:

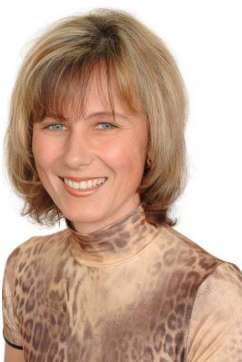

Prof. DSc. Tetiana Bubela

Education: Graduate Diploma in Information and Measuring Technique.

Professional interests: Practical research efforts are being steered towards the evolution of metrological technique for measuring the production quality characteristics. Author of over 90 scientific papers.

Lviv Polytechnic National University 12, Bandera str., Lviv 79008, Ukraine e-mail:paholuk@ukr.net

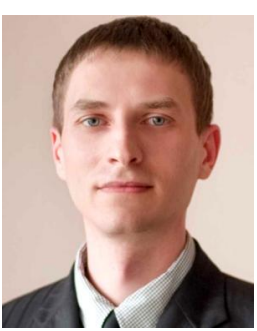

\section{Docent PhD, Yurij Yatsuk}

Docent of Lviv Polytechnic National University. He specializes in the field of automatized of technological process, control systems and measuring of electrical and non-electrical quantities. He is the author of over 30 scientific and methodical works, co-author of 1 textbook.

Lviv Polytechnic National University 12, Bandera str., Lviv 79008, Ukraine e-mail:jazuk.jurij@gmail.com

\section{ACKNOWLEDGEMENT}

The scientific results, presented in this article, were obtained within the frame of research project number 0115U000446, 01.01.2015-31.12.2017, financially supported by the Ministry of Education and Science of Ukraine. 